\title{
Necrotizing Soft-Tissue Infections: Current Concepts and Challenges
}

\author{
Avgoustou $\mathrm{C}^{1 *}$ and Avgoustou $\mathrm{Ch}^{2}$ \\ ${ }^{1}$ Department of Surgery, General Hospital of Nea Ionia Constantopoulion-Patission "Aghia Olga", Greece \\ ${ }^{2}$ Department of Pathology, Attikon University Hospital, National and Kapodistrian University of Athens Medical \\ School, Greece
}

\section{Editorial}

Necrotizing Soft-Tissue Infection (NSTI) and the most fulminant form of it, Necrotizing Fasciitis (NF), are rather uncommon but severe and destructive infectious diseases with high rates of mortality. Yearly incidence is reported to be 0.4 cases per 100,000 habitats, while reported mortality ranges from $21 \%$ to $43 \%$ [1]. Any part of the body can be affected, but perineum, extremities and trunk appear to be the most common areas. Advanced age, shock upon admission, host's comorbidities or immunosuppression are risk factors related with higher rates of mortality. Diabetics are notably more susceptible to the disease, and particularly to a more delayed presentation, a more extended disease and a more frequent unfavorable outcome. Delay to treatment is identified as a central co-factor for mortality as is the only modifiable parameter. NSTI and the NF describe a group of heterogenous, fulminant infections, mostly polymicrobial that spread through fascial planes and cause necrosis of skin, soft tissues and muscles. Systemic manifestations are a common feature of the prolonged disease, representing the resulting Systemic Inflammatory Response Syndrome (SIRS), septic shock or Multiple Organ Dysfunction Syndrome (MODS) or the effect of exotoxins, which are released from commonly involved bacteria, such as Group A Streptococci (GAS), Methicillin-Resistant Staphylococcus Aureus (MRSA) and Clostridium perfringens. The infection usually originates from the gastrointestinal or genitourinary tract or a dermal portal of entry. A traumatic injury is commonly identified as the key point for bacterial impregnation to the infectious focus. From there on, initiation of the cytokine cascade leads to endothelial damage and, further, to microthromboses of vessels feeding the fascia as well as to prolonged local vasoconstriction and leukocyte infiltration, all synergically leading to ischemic necrosis and liquefaction of the fascias and the surrounding tissues [1,2]. Patient's presentation can range from subtle physical examination findings to systemic signs of sepsis. Early stages may present only minimal local findings, including pain out of proportion to physical examination, erythema or skin discoloration, local warmth, tense edema beyond erythematous area, induration and numbness. Once the infection progresses, "hard signs" can be seen, including severe pain or local anesthesia, blistering and hemorrhagic bullae, foul odor, purulent collections, skin sloughing or necrosis, crepitus/soft tissue emphysema, as well as systemic manifestations. The interval between the onset of symptoms and initiation of treatment is marked as the crucial determinant for outcome. Patients may present late, as a consequence of the rapid progress of the pathological condition, the lack of impressive overlying skin changes and the limited degree of pain that results from the NF associated necrosis of superficial nerves. On the other hand, recently, in clinical practice, time to surgical intervention decreases, reflecting the increasing awareness and concern for NSTIs. An NSTI should be suspected in those patients with a soft tissue infection who rapidly deteriorate. However, the diagnosis can be challenging in that it may be difficult to distinguish between the more common non-necrotizing soft-tissue infections (i.e., cellulitis) and the early NSTIs without pathognomonic signs, and in that it might be existing a profound discrepancy between the little or absent overlying skin changes and the progressed underlying infectious disease. Expected laboratory results include leukocytosis (leukopenia in immunocompetents), elevated C-reactive protein, hyperglycemia especially among diabetics, high urea due to dehydration, hyponatremia, low calcium due to deposition to necrotic subcutaneous fat, elevated creatinine level when kidney injury has occurred and CK elevation in cases complicated with myositis. CT scan is suggested for diagnosis in equivocal cases and for assessment of infection's extent and severity, but should never delay surgical exploration of the critical patient. Utility of existing scores (i.e. LRINEC) designed to validate the diagnosis is contested, being only adjuncts supporting the clinical diagnosis. An integrated and multidisciplinary management is mandatory to

(2021 Avgoustou C. Th Attribution License, which permits unrestricted use, distribution, and reproduction in any medium, provided the original work is properly cited. 
a favorable outcome. Broad spectrum antibiotics near presentation covering for Gram positive, Gram negative and anaerobes, blood cultures before first dose, a laboratory work-up and aerometry, as well as variable supporting, intensive resuscitation and correction of metabolic disturbances when needed, account for the prompt firstline treatment the patient should receive in a short time frame, simultaneously to the preparation for transition to the operating room. The single most important determinant of survival is immediate surgical wide debridement. For indistinct physical findings, an incisional biopsy for rapid tissue frozen section and/or gram stain can be achieved before excision. On surgical exploration, all necrotic and ischemic tissues and even tissues of doubtful viability should be excised, until healthy tissue is exposed, and sent for histologycultures. Tissue planes should be opened, debrided, cleansed and drained. In those patients with extended perineal infection a diverting colostomy should be fashioned to control contamination of the residual wound. Testicles are usually spared even in extensive perineal disease, because of their independent blood supply. Compartment syndrome can be a common complication of lower extremities' NF, but amputation is rarely needed if timely referral of NSTI patients is achieved. Operative findings validating NF include dull grey (necrotic) fascias-tissues, small vessel thrombosis(lack of bleeding), tissues easily elevated off of the fascia (positive "finger test"), nonconstructing muscle and "dishwater" pus [1]. Bacteria commonly retrieved include Hemolytic streptococcus, especially GAS, Staphylococcus aureus or haemolyticus or MRSA, Enterobacteriaceae (E. coli, Pseudomonas aeruginosa, Acinetobacter sp., Klebsiella sp.), Bacteroides sp., Peptostreptococcus, Clostridium perfringens, Proteus mirabilis, Fusobacterium sp. and Citrobacter freundi. Survival after NSTI or NF attack is characterized by prolonged hospital stay, increased financial burden and need for collaboration of many specialties. For the immediate postoperative period, intensive care unit or high dependency unit monitoring is essential, aiming to restoration of intravascular volume and adequate end-organ perfusion and tissue oxygenation. Blood transfusion, coagulopathy correction and hemodialysis may be needed, depending on the individual patient's requirements. Adjunct therapies such as the IV immunoglobulin (binds and inactivates the circulating super antigens) and the use of hyperbaric oxygen (enhances oxygen delivery), despite of their high cost and relative unavailability, they have been found not providing certain benefit on survival [1]. Modulation and de-escalation to a more targeted therapy contribute to patient's improved outcome. Dramatic improvement is detected only if sepsis and source infection is controlled, primarily after a prompt and adequate debridement. However, practice guidelines for best management of NTSI/NF after initial necrectomy/debridement and antibiotics do not exist. Wound re exploration should be warranted within $24 \mathrm{~h}$ to assess the need for further incisions and excisions. Close wound care, namely serial dressing changes and frequent estimates of surgical wounds and tissue viability twice or more daily, are required for the first $72 \mathrm{~h}$. Topical negative pressure is reported to reduce wound surface, extract wound exudates, improve perfusion and cell acceleration and induce granulation and thus, can be largely used for non-perineal defects. Enteral feeding, vitamins A, $\mathrm{C}$ and minerals complement patient's care. Targeted antibiotics should be administered for 3 to 5 days after the systemic signs and symptoms and the most local signs of soft-tissue infection have been resolved. Reconstruction of residual defects by secondary intention or using skin graft or flaps are the more commonly used options, and are guided by the individual patient's requirement [2]. Concluding, a soft tissue infection with sudden onset, that is spreading and deteriorating rapidly, accompanied or not by determinable systemic signs, should set the suspicion for NSTI and requires immediate surgical assessment. A meticulous physical examination and a clear understanding of the disease's history, can frequently establish the diagnosis. Surgery should never be delayed, because septic state reversal requires all necrotic and infected tissues to be timely removed.

\section{References}

1. Garcia NM, Cai J. Aggressive soft tissue infections. Surg Clin North Am. 2018 ;98(5):1097-108.

2. Cocanour CS, Chang P, Huston JM. Management and novel adjuncts of necrotizing soft tissue infections. Surg Infect (Larchmt). 2017;18(3):25072. 